\title{
On convergence of two-stage iterative scheme
}

\author{
Vaibhav Shekhar ${ }^{1} \cdot$ Chinmay Kumar Giri ${ }^{2} \cdot$ Debasisha Mishra $^{1}$
}

Received: 20 May 2020 / Accepted: 8 January 2021 / Published online: 6 February 2021

(C) Forum D'Analystes, Chennai 2021

\begin{abstract}
Climent and Perea [Journal of Computational and Applied Mathematics 58:43-48, 2003; MR2013603] proposed first the convergence theory of two-stage iterative scheme for solving real rectangular linear systems. In this article, we revisit the same theory. The first main result provides some sufficient conditions which guarantee that the induced splitting from a two-stage iterative scheme is a proper weak regular splitting. We then establish a few comparison results. Out of these, many are even new in nonsingular matrix setting. Further, we study the monotone convergence theory of the two-stage iterative method. Besides these, we also prove the uniqueness of a proper splitting of a rectangular matrix under certain assumptions.
\end{abstract}

Keywords Linear system · Moore-Penrose inverse - nonnegativity · Proper splitting $\cdot$ Two-stage iterative method $\cdot$ Comparison theorem

Mathematics Subject Classification 15A09

Communicated by Samy Ponnusamy.

Debasisha Mishra

kapamath@gmail.com

Vaibhav Shekhar

vaibhavshekhar29@gmail.com

Chinmay Kumar Giri

ckg2357@gmail.com

1 Department of Mathematics, National Institute of Technology Raipur, Raipur,

Chhattisgarh 492010, India

2 Department of Mathematics, Seemanta Engineering College, Jharpokharia, Odisha 757 086, India 


\section{Introduction}

Consider the problem of finding a numerical solution of a linear system of the form

$$
A x=b,
$$

where $A \in \mathbb{R}^{m \times n}$ is large and sparse, and $b \in \mathbb{R}^{m}$. Iterative methods are more useful for solving such equations as it avoids the use of the normal system $A^{T} A x=A^{T} b$, where $A^{T} A$ is frequently ill-conditioned and influenced greatly by roundoff errors (see [12]). Here, $A^{T}$ denotes the transpose of the matrix $A$. The linear system (1.1) appears in many scientific and engineering problems. In particular, such a system appears in deconvolution problems with a smooth kernel. Singular linear systems also appear in finite difference representation of Neumann problems (see [22]). Berman and Plemmons [4] introduced the following iterative scheme

$$
x_{k+1}=U^{\dagger} V x_{k}+U^{\dagger} b, \quad k=0,1,2, \ldots,
$$

to find an approximate solution of the system (1.1) using a proper splitting $A=U-V$, where $U^{\dagger}$ denotes the Moore-Penrose inverse of $U$. A splitting $A=$ $U-V$ of $A \in \mathbb{R}^{m \times n}$ is called a proper splitting [4] if $R(U)=R(A)$ and $N(U)=N(A)$, where $R(B)$ and $N(B)$ denote the range space and the null space of a matrix $B$, respectively. The authors of [4] showed that the iterative scheme (1.2) converges to $A^{\dagger} b$, the least-squares solution of minimum norm of (1.1) for every initial vector $x_{o}$ if and only if $\rho\left(U^{\dagger} V\right)<1$ (see Corollary 1, [4]). Here, $\rho(B)$ denotes the spectral radius of a matrix $B$.

Later on, many authors introduced different sub-classes of proper splittings and studied their convergence theory (see [9, 19]). However, the classical iterative methods are computationally expensive, which attracts the researcher to develop fast iterative solvers. In this context, Nichols [21] proposed the notion of the twostage iterative method to find an iterative solution of a nonsingular linear system. Lanzkron et al. [15] and, Frommer and Szyld [11] then studied further its convergence theory. The convergence of two-stage iterative scheme in case of positive definite linear systems is studied extensively in [6, 8, 16, 17], while in [7] and [26], the authors considered the singular linear systems. Climent and Perea [10] generalized the notion of the two-stage iterative technique to rectangular matrix case that is recalled next. Let us consider an iterative scheme of the form

$$
U x_{k+1}=V x_{k}+b,
$$

where $A=U-V$ is a proper splitting of $A$ for solving a consistent linear system of the form (1.1). The scheme (1.3) is called outer iteration. At each step of (1.3), we must solve the inner equations

$$
U y=\tilde{b}, \quad \tilde{b}=V x_{k}+b .
$$

Solving (1.4) by another iterative scheme (called inner iteration) which is formed by using a proper splitting $U=F-G$ that performs $s(k)$ inner iterations, we get the two-stage iterative scheme [10], i.e., 


$$
x_{k+1}=\left(F^{\dagger} G\right)^{s(k)} x_{k}+\sum_{j=0}^{s(k)-1}\left(F^{\dagger} G\right)^{j} F^{\dagger}\left(V x_{k}+b\right), \quad k=1,2, \ldots,
$$

where $s(k)$ is the number of inner iteration which depends on $k$. We say that a twostage iterative scheme (1.5) is stationary when $s(k)=s$ for all $k$, while it is called non-stationary when $s(k)$ changes with $k$. If $A=U-V$ is a proper regular splitting and $U=F-G$ is a proper weak regular splitting of type I (see Definition 2.4), the authors of [10] established that the iterative scheme (1.5) converges to $A^{\dagger} b$, the minimum norm least-squares solution of system (1.1) for any initial vector $x_{o}$. In this article, we further establish the proper regularity of the induced splitting induced from the two-stage iterative scheme (1.5) and obtain various comparison results for the faster convergence of the two-stage iterative scheme. Motivation of the current work comes from the results of Bai and Wang [1], and Wang and Zhao [25]. Although most of our results in this paper are for the rectangular linear system, many results are even new in the nonsingular matrix setting and are stated as corollaries.

The structure of this paper is as follows. In Sect. 2, we introduce some notations and definitions which are helpful in proving the main results. The uniqueness of a proper splitting for a certain class of real rectangular matrices is also proved in Sect. 2. After revisiting the two-stage iterative scheme and its convergence criteria established in [10], we analyze the proper regularity of the induced splitting by the two-stage iterative scheme and derive a few comparison theorems of different splittings for two-stage iterative schemes in Sect. 3. In Sect. 4, we set up the monotone convergence theorem for a stationary two-stage iterative scheme.

\section{Preliminaries}

Throughout this paper, all matrices considered are real, and $\mathbb{R}^{m \times n}$ denotes the set of all real $m \times n$ matrices. A matrix $A \in \mathbb{R}^{m \times n}$ is called nonnegative(positive), if each entry of $A$ is nonnegative(positive), and is denoted by $A \geq 0(A>0)$. For $B, C \in \mathbb{R}^{m \times n}$, we write $B \geq C$ if $B-C \geq 0$. The spectral radius of a matrix $A \in$ $\mathbb{R}^{n \times n}$ is equal to the maximum of the moduli of the eigenvalues of $A$. Next, we recall a few results that deal with nonnegativity of a matrix and the spectral radius.

Theorem 2.1 (Theorem 2.20, [24])

Let $A \in \mathbb{R}^{n \times n}$ and $A \geq 0$. Then

(i) A has a nonnegative real eigenvalue equal to its spectral radius.

(ii) To $\rho(A)$, there corresponds an eigenvector $x \geq 0$.

Theorem 2.2 (Theorem 3.15, [24])

If $B \in \mathbb{R}^{n \times n}$ with $\rho(B)<1$, then $I-B \quad$ is nonsingular, and $(I-B)^{-1}=I+B+B^{2}+\cdots$, the series on the right converging. Conversely, if the series on the right converges, then $\rho(B)<1$. 
A nonempty subset $K$ of $\mathbb{R}^{n}$ is called a convex cone if $\lambda \geq 0$ implies $\lambda K \subseteq K$ and $K+K \subseteq K$, pointed if $K \cap-K \neq \phi$, and solid if interior of $K$ is nonempty. A pointed, solid, closed convex cone is called a full cone. The next result is about a full cone in $\mathbb{R}^{n}$.

\section{Lemma 2.3 (Lemma 1, [4])}

Let $K$ be a full cone in $\mathbb{R}^{n}$ and $\left\{s_{i}\right\}_{i=0}^{\infty}$ be a $K$-monotone non-decreasing sequence. Let $t \in \mathbb{R}^{n}$ be such that $t-s_{i} \in K$ for every positive integer $i$. Then the sequence $\left\{s_{i}\right\}_{i=0}^{\infty}$ converges.

If $L \oplus M=\mathbb{R}^{n}$, then $P_{L, M}$ is referred as the projection onto $L$ along $M$. So, $P_{L, M} A=A$ if and only if $R(A) \subseteq L$, and $A P_{L, M}=A$ if and only if $N(A) \supseteq M$. If $L \perp M$, then $P_{L, M}$ is denoted by $P_{L}$. For $A \in \mathbb{R}^{m \times n}$, the unique matrix $X \in \mathbb{R}^{n \times m}$ is called the Moore-Penrose inverse of $A$ if it satisfies the following four equations:

$$
A X A=A, \quad X A X=X, \quad(A X)^{T}=A X \operatorname{and}(X A)^{T}=X A,
$$

and is denoted by $A^{\dagger}$. It always exists. When $A$ is nonsingular, then the MoorePenrose inverse of $A$ coincides with the usual matrix inverse, i.e., $A^{\dagger}=A^{-1}$. A matrix $A \in \mathbb{R}^{m \times n}$ is called semimonotone if $A^{\dagger} \geq 0$ (see [5]). A few properties of $A^{\dagger}$ which will be frequently used in this paper are: $R\left(A^{\dagger}\right)=R\left(A^{T}\right) ; N\left(A^{\dagger}\right)=$ $N\left(A^{T}\right) ; A A^{\dagger}=P_{R(A)}$ and $A^{\dagger} A=P_{R\left(A^{T}\right)}$ (see [2] for more details). Further, if $A$ and $B$ are such that the matrix product $A B$ is defined, then $(A B)^{\dagger}=B^{\dagger} A^{\dagger}$ if and only if $A^{\dagger} A B B^{T} A^{T}=B B^{T} A^{T}$ and $B B^{\dagger} A^{T} A B=A^{T} A B$ (see [13]). The following theorem that is a part of Theorem 1, [4] collects some properties of a proper splitting.

Theorem 2.4 (Theorem 1, [4])

Let $A=U-V$ be a proper splitting of $A \in \mathbb{R}^{m \times n}$. Then

(i) $A=U\left(I-U^{\dagger} V\right)$,

(ii) $I-U^{\dagger} V$ is nonsingular,

(iii) $\quad A^{\dagger}=\left(I-U^{\dagger} V\right)^{-1} U^{\dagger}$.

In 2018, Mishra and Mishra [20] proved the uniqueness of proper splitting for a given real square matrix (see Theorem 2.2, [20]) under the assumption of a few conditions. We next extend the same result to real rectangular matrices.

Theorem 2.5 Let $A \in \mathbb{R}^{m \times n}$. If there is a real $n \times n$ matrix $H$ such that $R(H) \subseteq$ $R\left(A^{T}\right), N(A) \subseteq N(H)$ and $\rho(H)<1$, then $A=U-V$ is a unique proper splitting and $H=U^{\dagger} V$.

Proof Let $U=A(I-H)^{-1}$. Then $R(U)=R(A)$. Let $x \in N(U)$. Then $0=U x=$ $A(I-H)^{-1} x$. Now, pre-multiplying $A^{\dagger}$ to this, we get 


$$
\begin{aligned}
0 & =A^{\dagger} A(I-H)^{-1} x \\
& =A^{\dagger} A\left(I+H+H^{2}+\cdots\right) x \\
& =\left(A^{\dagger} A+A^{\dagger} A H+A^{\dagger} A H^{2}+\cdots\right) x \\
& =\left(A^{\dagger} A+H+H^{2}+\cdots\right) x \\
& =\left(A^{\dagger} A+H A^{\dagger} A+H^{2} A^{\dagger} A+\cdots\right) x \\
& =\left(I+H+H^{2}+\cdots\right) A^{\dagger} A x \\
& =(I-H)^{-1} A^{\dagger} A x .
\end{aligned}
$$

Again, pre-multiplying $A(I-H)$ to the above expression, we get $A x=0$, which implies $N(U) \subseteq N(A)$. Let us now consider $x \in N(A)$. Then $0=A x=U(I-$ $H) x=U x-U H x=U x$. Hence, $N(U)=N(A)$. Next, we prove $U^{\dagger}=(I-H) A^{\dagger}$. To do this, we need to verify that $A^{\dagger} A(I-H)^{-1}\left((I-H)^{-1}\right)^{T} A^{T}=(I-H)^{-1}((I-$ $\left.H)^{-1}\right)^{T} A^{T} \quad$ and $\quad(I-H)^{-1}(I-H) A^{T} A(I-H)^{-1}=A^{T} A(I-H)^{-1}$. Clearly, $(I-H)^{-1}(I-H) A^{T} A(I-H)^{-1}=A^{T} A(I-H)^{-1}$. Using the fact that $A^{\dagger} A(I-$ $H)^{-1}=(I-H)^{-1} A^{\dagger} A$ and $\left(A^{\dagger} A\right)^{T}=A^{\dagger} A$, we have

$$
\begin{aligned}
A^{\dagger} A(I-H)^{-1}\left((I-H)^{-1}\right)^{T} A^{T} & =(I-H)^{-1} A^{\dagger} A\left((I-H)^{-1}\right)^{T} A^{T} \\
& =(I-H)^{-1}\left(A(I-H)^{-1} A^{\dagger} A\right)^{T} \\
& =(I-H)^{-1}\left(A A^{\dagger} A(I-H)^{-1}\right)^{T} \\
& =(I-H)^{-1}\left(A(I-H)^{-1}\right)^{T} \\
& =(I-H)^{-1}\left((I-H)^{-1}\right)^{T} A^{T} .
\end{aligned}
$$

Thus, $\quad U^{\dagger}=(I-H) A^{\dagger} \quad$ and $\quad U^{\dagger} V=U^{\dagger}(U-A)=U^{\dagger} U-U^{\dagger} A=A^{\dagger} A-(I-$ $H) A^{\dagger} A=H A^{\dagger} A=H$. For the uniqueness, let $A=U_{1}-V_{1}$ be another proper splitting such that $H=U_{1}^{\dagger} V_{1}$. Then $U_{1} H=V_{1}=U_{1}-A$ and thus $U_{1}=A(I-H)^{-1}=U$.

We refer the reader to $[3,19]$ for different methods of construction of a proper splitting. We next recall the definitions of two sub-classes of proper splittings.

Definition 2.6 ([14])

A proper splitting $A=U-V$ of $A \in \mathbb{R}^{m \times n}$ is called a

(i) Proper regular splitting if $U^{\dagger} \geq 0$ and $V \geq 0$,

(ii) Proper weak regular splitting if $U^{\dagger} \geq 0$ and $U^{\dagger} V \geq 0$.

The next result combines the convergence criteria for both the above classes of proper splittings (see Theorem 1.3, [14] and Corollary 4, [4]).

Theorem 2.7 Let $A=U-V$ be any of the above class of splittings of $A \in \mathbb{R}^{m \times n}$. Then $A$ is semimonotone if and only if $\rho\left(U^{\dagger} V\right)<1$. 
A comparison of the spectral radii of two iterative schemes arising from two proper splittings is useful for improving the speed of convergence of the iteration scheme (1.2). In this direction, several comparison results have been introduced in the literature both for rectangular and nonsingular matrices. Recently, Mishra and Mishra [20] proved the following comparison result.

Theorem 2.8 (Theorem 2.8, [20])

Let $A=U_{1}-V_{1}$ be a proper weak regular splitting and $A=U_{2}-V_{2}$ be a proper regular splitting of a semimonotone matrix $A \in \mathbb{R}^{m \times n}$. If $U_{1}^{\dagger} \geq U_{2}^{\dagger}$, then $\rho\left(U_{1}^{\dagger} V_{1}\right) \leq \rho\left(U_{2}^{\dagger} V_{2}\right)<1$.

We now recall another comparison result proved by Mishra [18].

Theorem 2.9 (Theorem 3.4, [18])

Let $A=U_{1}-V_{1}=U_{2}-V_{2}$ be two proper weak regular splittings of a semimonotone matrix $A \in \mathbb{R}^{m \times n}$. If either of the following cases holds,

(i) $V_{2} \geq V_{1}$,

(ii) $\quad U_{1}^{\dagger} \geq U_{2}^{\dagger}, V_{1} \geq 0$, then $\rho\left(U_{1}^{\dagger} V_{1}\right) \leq \rho\left(U_{2}^{\dagger} V_{2}\right)<1$.

\section{Two-stage iterative method}

In this section, we present some sufficient conditions for the convergence of two stage-iterative schemes and then obtain comparison of the spectral radii of two different iteration matrices arising out of two matrix splittings which is useful for improving the speed of the two-stage iterative scheme. For convenience, let us rewrite the two-stage iterative scheme introduced by Climent and Perea [10] as

$$
x_{k+1}=\left(F^{\dagger} G\right)^{s(k)} x_{k}+\sum_{j=0}^{s(k)-1}\left(F^{\dagger} G\right)^{j} F^{\dagger}\left(V x_{k}+b\right), \quad k=1,2, \ldots .
$$

For a given initial vector $x_{o}$, the two-stage iterative scheme (3.1) produces the sequence of vectors

$$
x_{k+1}=T_{s(k)} x_{k}+P_{s(k)}^{\dagger} b, \quad k=0,1,2, \ldots,
$$

where

$$
T_{s(k)}=\left(F^{\dagger} G\right)^{s(k)}+\sum_{j=0}^{s(k)-1}\left(F^{\dagger} G\right)^{j} F^{\dagger} V \quad \text { and } \quad P_{s(k)}^{\dagger}=\sum_{j=0}^{s(k)-1}\left(F^{\dagger} G\right)^{j} F^{\dagger}
$$

For stationary two-stage iteration scheme, Climent and Perea [10] proved the following convergence result.

Theorem 3.1 (Theorem 3, [10])

Let $A=U-V$ be a proper regular splitting of a semimonotone matrix 
$A \in \mathbb{R}^{m \times n}$. Let $U=F-G$ be a proper weak regular splitting. Then the stationary two-stage iterative scheme is convergent for any initial vector $x_{o}$.

For non-stationary two-stage scheme, we have the following result that generalizes Theorem 4.4, [11]. The proof is similar to Theorem 3, [10], therefore we omit it.

Theorem 3.2 Let $A=U-V$ be a proper regular splitting of a semimonotone matrix $A \in \mathbb{R}^{m \times n}$. Let $U=F-G$ be a proper weak regular splitting. Then the nonstationary two-stage iterative method (3.1) is convergent for any sequence $s(k) \geq 1, k=0,1,2, \ldots$.

The next result shows that the iteration matrix $T_{s(k)}$ induces a unique proper weak regular splitting $A=P_{s(k)}-P_{s(k)} T_{s(k)}$ under a few sufficient conditions.

Theorem 3.3 Let $A=U-V$ be a proper regular splitting of a semimonotone matrix $A \in \mathbb{R}^{m \times n}$. Let $U=F-G$ be a proper weak regular splitting. Then the unique splitting $A=P_{s(k)}-P_{s(k)} T_{s(k)}$ induced by the iteration matrix $T_{s(k)}$ is a proper weak regular splitting.

Proof From (3.3), we have

$$
T_{s(k)}=\left(F^{\dagger} G\right)^{s(k)}+\sum_{j=0}^{s(k)-1}\left(F^{\dagger} G\right)^{j} F^{\dagger} V=I-\left(I-\left(F^{\dagger} G\right)^{s(k)}\right)\left(I-U^{\dagger} V\right)
$$

and

$$
P_{s(k)}^{\dagger}=\sum_{j=0}^{s(k)-1}\left(F^{\dagger} G\right)^{j} F^{\dagger}=\left(I-\left(F^{\dagger} G\right)^{s(k)}\right)\left(I-F^{\dagger} G\right)^{-1} F^{\dagger}=\left(I-\left(F^{\dagger} G\right)^{s(k)}\right) U^{\dagger} .
$$

Let $X=U\left(I-\left(F^{\dagger} G\right)^{s(k)}\right)^{-1}$. Then $X P_{s(k)}^{\dagger}=U U^{\dagger}$ and $P_{s(k)}^{\dagger} X=U^{\dagger} U$. So, $X P_{s(k)}^{\dagger}$ and $P_{s(k)}^{\dagger} X$ are symmetric. Now, $P_{s(k)}^{\dagger} X P_{s(k)}^{\dagger}=P_{s(k)}^{\dagger}$ and $X P_{s(k)}^{\dagger} X=X$. Hence $X=\left(P_{s(k)}^{\dagger}\right)^{\dagger}=P_{s(k)}=U\left(I-\left(F^{\dagger} G\right)^{s(k)}\right)^{-1}$. Clearly, $R\left(P_{s(k)}\right)=R(U)$. It remains to show that $N\left(P_{s(k)}\right)=N(U)$. We can see that

$$
\begin{aligned}
& P_{s(k)} x=0 \\
\Leftrightarrow & U\left(I-\left(F^{\dagger} G\right)^{s(k)}\right)^{-1} x=0 \\
\Leftrightarrow & \left(I-\left(F^{\dagger} G\right)^{s(k)}\right) U^{\dagger} U\left(I-\left(F^{\dagger} G\right)^{s(k)}\right)^{-1} x=0 \\
\Leftrightarrow & U^{\dagger} U\left(I-\left(F^{\dagger} G\right)^{s(k)}\right)\left(I-\left(F^{\dagger} G\right)^{s(k)}\right)^{-1} x=0 \\
\Leftrightarrow & U^{\dagger} U x=0 \\
\Leftrightarrow & U x=0 .
\end{aligned}
$$

Hence $N\left(P_{s(k)}\right)=N(U)$. Now, we have 


$$
\begin{aligned}
P_{s(k)}^{\dagger} P_{s(k)} T_{s(k)} & =P_{s(k)}^{\dagger} P_{s(k)}\left(F^{\dagger} G\right)^{s(k)}+P_{s(k)}^{\dagger} P_{s(k)}\left(\sum_{j=0}^{s(k)-1}\left(F^{\dagger} G\right)^{j} F^{\dagger} V\right) \\
& =U^{\dagger} U\left(F^{\dagger} G\right)^{s(k)}+U^{\dagger} U\left(\sum_{j=0}^{s(k)-1}\left(F^{\dagger} G\right)^{j} F^{\dagger} V\right) \\
& =\left(F^{\dagger} G\right)^{s(k)}+\sum_{j=0}^{s(k)-1}\left(F^{\dagger} G\right)^{j} F^{\dagger} V \\
& =T_{s(k)} \geq 0 .
\end{aligned}
$$

Then, it holds that $P_{s(k)}-P_{s(k)} T_{s(k)}=P_{s(k)}\left(I-T_{s(k)}\right)=U\left(I-\left(F^{\dagger} G\right)^{s(k)}\right)^{-1}(I-$ $\left.\left(F^{\dagger} G\right)^{s(k)}\right)\left(I-U^{\dagger} V\right)=U\left(I-U^{\dagger} V\right)=A \quad$ by $\quad$ Theorem $\quad$ 2.4. So $P_{s(k)}^{\dagger}=$ $\sum_{j=0}^{s(k)-1}\left(F^{\dagger} G\right)^{j} F^{\dagger} \geq 0$ and $P_{s(k)}^{\dagger} P_{s(k)} T_{s(k)} \geq 0$ implies that the induced splitting $A=$ $P_{s(k)}-P_{s(k)} T_{s(k)}$ is a proper weak regular splitting.

For uniqueness, suppose that $T_{s(k)}$ induces another splitting $A=R_{s(k)}-R_{s(k)} T_{s(k)}$. Then $R_{s(k)}=A\left(I-T_{s(k)}\right)^{-1}=P_{s(k)}$. Hence, the proper splitting $A=P_{s(k)}-$ $P_{s(k)} T_{s(k)}$ induced by the iteration matrix $T_{s(k)}$ is unique.

We provide below sufficient conditions which guarantee that the induced splitting from the two-stage iterative scheme is a proper regular one.

Theorem 3.4 Let $A=U-V$ be a proper regular splitting of a semimonotone matrix $A \in \mathbb{R}^{m \times n}$. Let $U=F-G$ be a proper regular splitting. If $\left(F^{\dagger} G\right)^{2} \leq F^{\dagger} G$, then the two-stage splitting $A=P_{s(k)}-P_{s(k)} T_{s(k)}$ is a proper regular splitting.

Proof We have

$$
\begin{aligned}
P_{s(k)} T_{s(k)} & =P_{s(k)}-A \\
& =U\left(I-\left(F^{\dagger} G\right)^{s(k)}\right)^{-1}-U+V \\
& =U\left(\left(I-\left(F^{\dagger} G\right)^{s(k)}\right)^{-1}-I\right)+V \\
& =U\left(F^{\dagger} G\right)^{s(k)}\left(I-\left(F^{\dagger} G\right)^{s(k)}\right)^{-1}+V .
\end{aligned}
$$

Now, we only need to show that $U\left(F^{\dagger} G\right)^{s(k)} \geq 0$. It is enough to prove that $U\left(F^{\dagger} G\right)^{2} \geq 0$.

$$
\begin{aligned}
U\left(F^{\dagger} G\right)^{2} & =(F-G) F^{\dagger} G F^{\dagger} G \\
& =\left(F F^{\dagger} G-G F^{\dagger} G\right) F^{\dagger} G \\
& =\left(G-G F^{\dagger} G\right) F^{\dagger} G \\
& =G F^{\dagger} G-G\left(F^{\dagger} G\right)^{2} \\
& \geq 0 .
\end{aligned}
$$

As $F^{\dagger} G \geq 0$ and $U\left(F^{\dagger} G\right)^{2} \geq 0$, thus $U\left(F^{\dagger} G\right)^{s(k)} \geq 0$. Hence $P_{s(k)} T_{s(k)} \geq 0$. 
The example given below demonstrates the above theorem.

Example 3.5 Let us consider a semimonotone matrix $A$ of the form $A=\left[\begin{array}{ccc}1 & 1 & 0 \\ 0 & 0 & 9 \\ 10 & 10 & 0 \\ 0 & 0 & 2\end{array}\right]$. Setting $U=\left[\begin{array}{ccc}1 & 1 & 0 \\ 0 & 0 & 18 \\ 10 & 10 & 0 \\ 0 & 0 & 4\end{array}\right]$, we get a proper regular splitting of $A$. Further, if $F=\left[\begin{array}{ccc}1 & 1 & 0 \\ 0 & 0 & 24.3 \\ 10 & 10 & 0 \\ 0 & 0 & 5.4\end{array}\right]$, then $U=F-G$ is a proper regular splitting of $U$. Clearly, $F^{\dagger} G=\left[\begin{array}{ccc}0 & 0 & 0 \\ 0 & 0 & 0 \\ 0 & 0 & 0.2593\end{array}\right] \geq\left[\begin{array}{ccc}0 & 0 & 0 \\ 0 & 0 & 0 \\ 0 & 0 & 0.0672\end{array}\right]=\left(F^{\dagger} G\right)^{2}$. Also,

$$
\begin{aligned}
P_{S(k)}^{\dagger}= & {\left[\begin{array}{cccc}
0.005 & 0 & 0.0495 & 0 \\
0.005 & 0 & 0.0495 & 0 \\
0 & 0.0494 & 0 & 0.011
\end{array}\right] \geq 0 } \\
P_{S(k)} T_{S(k)} & =\left[\begin{array}{ccc}
0 & 0 & 0 \\
0 & 0 & 10.2971 \\
0 & 0 & 0 \\
0 & 0 & 2.2882
\end{array}\right] \geq 0 \text {. Therefore, } A=P_{s(k)}-P_{s(k)} T_{s(k)} \text { is a proper }
\end{aligned}
$$
regular splitting.

The next result is motivated by Corollary 2.7, [25] which says that the conclusion of Theorem 3.4 can also be achieved if we replace the condition $\left(F^{\dagger} G\right)^{2} \leq F^{\dagger} G$ by $G F^{\dagger} G \leq G$.

Theorem 3.6 Let $A=U-V$ be a proper regular splitting of a semimonotone matrix $A \in \mathbb{R}^{m \times n}$. Let $U=F-G$ be a proper regular splitting. If $G F^{\dagger} G \leq G$, then the two-stage splitting $A=P_{s(k)}-P_{s(k)} T_{s(k)}$ is a proper regular splitting.

Proof By pre-multiplying $F^{\dagger}$ to the given condition $G F^{\dagger} G \leq G$, we get $\left(F^{\dagger} G\right)^{2} \leq F^{\dagger} G$. By Theorem 3.4, we thus have $P_{s(k)} T_{s(k)} \geq 0$.

If we replace the condition $\left(F^{\dagger} G\right)^{2} \leq F^{\dagger} G$ by $F^{\dagger} U F^{\dagger} \geq 0$ in Theorem 3.4, then the induced splitting is proper regular. This is shown next which extends Theorem 2.3, [25] to rectangular matrix case.

Theorem 3.7 Let $A=U-V$ be a proper regular splitting of a semimonotone matrix $A \in \mathbb{R}^{m \times n}$. Let $U=F-G$ be a proper regular splitting. If $F^{\dagger} U F^{\dagger} \geq 0$, then the two-stage splitting $A=P_{s(k)}-P_{s(k)} T_{s(k)}$ is a proper regular splitting.

Proof We have $0 \leq F^{\dagger} U F^{\dagger}=\left(I-F^{\dagger} G\right) U^{\dagger} U F^{\dagger}=\left(I-F^{\dagger} G\right) F^{\dagger}=F^{\dagger}-F^{\dagger} G F^{\dagger}$, i.e., $F^{\dagger} G F^{\dagger} \leq F^{\dagger}$. By post-multiplying $G$ to the expression $F^{\dagger} G F^{\dagger} \leq F^{\dagger}$, we obtain $\left(F^{\dagger} G\right)^{2} \leq F^{\dagger} G$. We thus have $P_{s(k)} T_{s(k)} \geq 0$, by Theorem 3.4. 
The problem of comparison of the convergence rate of two different two-stage iterative schemes is not studied much in the literature even for the nonsingular matrix case. One of the main objectives of this paper is to study this problem. The next three results are in this direction. The different two-stage iterative schemes are framed by taking two different proper splittings $U=\bar{F}-\bar{G}=\widehat{F}-\widehat{G}$ with corresponding iteration matrices as $\bar{T}_{s(k)}=\left(\bar{F}^{\dagger} \bar{G}\right)^{s(k)}+\sum_{j=0}^{s(k)-1}\left(\bar{F}^{\dagger} \bar{G}\right)^{j} \bar{F}^{\dagger} V$ and $\widehat{T}_{s(k)}=$ $\left(\widehat{F}^{\dagger} \widehat{G}\right)^{s(k)}+\sum_{j=0}^{s(k)-1}\left(\widehat{F}^{\dagger} \widehat{G}\right)^{j} F^{\dagger} V$ and with the same number of inner iterations $s(k)$.

Theorem 3.8 Let $A=U-V$ be a proper regular splitting of a semimonotone matrix $A \in \mathbb{R}^{m \times n}$. Let $U=\bar{F}-\bar{G}=\widehat{F}-\widehat{G}$ be proper weak regular splittings of $U$. If any one of the following conditions hold:

(i) $\bar{F}^{\dagger} \bar{G} \leq \widehat{F}^{\dagger} \widehat{G}$ and $\widehat{P}_{s(k)} \widehat{T}_{s(k)} \geq 0$,

(ii) $\quad \bar{F}^{\dagger} \bar{G} \leq \widehat{F}^{\dagger} \widehat{G}$ and $\bar{P}_{s(k)} \bar{T}_{s(k)} \geq 0$,

(iii) $\quad \bar{P}_{s(k)} \bar{T}_{s(k)} \leq \widehat{P}_{s(k)} \widehat{T}_{s(k)}$, then $\rho\left(\bar{T}_{s(k)}\right) \leq \rho\left(\widehat{T}_{s(k)}\right)<1$.

\section{Proof}

(i) By Theorem 3.2, we have $\rho\left(\bar{T}_{s(k)}\right)<1$ and $\rho\left(\widehat{T}_{s(k)}\right)<1$. By Theorem 3.3, the induced splittings $A=\bar{P}_{s(k)}-\bar{P}_{s(k)} \bar{T}_{s(k)}$ and $A=\widehat{P}_{s(k)}-\widehat{P}_{s(k)} \widehat{T}_{s(k)}$ are proper weak regular splittings. The condition $\widehat{P}_{s(k)} \widehat{T}_{s(k)} \geq 0$ yields that $A=$ $\widehat{P}_{s(k)}-\widehat{P}_{s(k)} \widehat{T}_{s(k)}$ is a proper regular splitting. Since $0 \leq \bar{F}^{\dagger} \bar{G} \leq \widehat{F}^{\dagger} \widehat{G}$, we have $\quad\left(\bar{F}^{\dagger} \bar{G}\right)^{s(k)} \leq\left(\widehat{F}^{\dagger} \widehat{G}\right)^{s(k)} \quad$ which implies $\quad\left[I-\left(\bar{F}^{\dagger} \bar{G}\right)^{s(k)}\right] U^{\dagger} \geq[I-$ $\left.\left(\widehat{F}^{\dagger} \widehat{G}\right)^{s(k)}\right] U^{\dagger}$ yielding $\bar{P}_{s(k)}^{\dagger} \geq \widehat{P}_{s(k)}^{\dagger}$. By Theorem 2.8 , we therefore obtain $\rho\left(\bar{T}_{s(k)}\right) \leq \rho\left(\widehat{T}_{s(k)}\right)$.

(ii) Using $\bar{F}^{\dagger} \bar{G} \leq \widehat{F}^{\dagger} \widehat{G}$, we get $\bar{P}_{s(k)}^{\dagger} \geq \widehat{P}_{s(k)}^{\dagger}$. Now, $\bar{P}_{s(k)} \bar{T}_{s(k)} \geq 0 \quad$ and $\bar{P}_{s(k)}^{\dagger} \geq \widehat{P}_{s(k)}^{\dagger}$ imply $\rho\left(\bar{T}_{s(k)}\right) \leq \rho\left(\widehat{T}_{s(k)}\right)$ by Theorem 2.9(ii).

(iii) Applying Theorem 2.9(i) to the induced weak regular splittings $A=$ $\bar{P}_{s(k)}-\bar{P}_{s(k)} \bar{T}_{s(k)}$ and $A=\widehat{P}_{s(k)}-\widehat{P}_{s(k)} \widehat{T}_{s(k)}$, we directly obtain $\rho\left(\bar{T}_{s(k)}\right) \leq \rho\left(\widehat{T}_{s(k)}\right)$.

The following example illustrates the above theorem.

Example 3.9 Let us consider a semimonotone matrix $A$ of the form $A=\left[\begin{array}{lll}1 & 0 & 1 \\ 0 & 1 & 0 \\ 1 & 0 & 1 \\ 2 & 0 & 2\end{array}\right]$. Setting $U=\left[\begin{array}{ccc}1.41 & 0 & 1.41 \\ 0 & 1.41 & 0 \\ 1.41 & 0 & 1.41 \\ 2.82 & 0 & 2.82\end{array}\right]$, we get a proper regular 
splitting $A=U-V$ of $A$. Further, if $\bar{F}=\left[\begin{array}{ccc}1.692 & 0 & 1.692 \\ 0 & 1.692 & 0 \\ 1.692 & 0 & 1.692 \\ 3.384 & 0 & 3.384\end{array}\right]$ and $\widehat{F}=\left[\begin{array}{ccc}3.243 & 0 & 3.243 \\ 0 & 3.243 & 0 \\ 3.243 & 0 & 3.243 \\ 6.486 & 0 & 6.486\end{array}\right]$, then $U=\bar{F}-\bar{G}=\widehat{F}-\widehat{G}$ are two proper weak regular splittings $\quad$ of $\quad U . \quad$ Clearly, $\bar{F}^{\dagger} \bar{G}=\left[\begin{array}{ccc}0.0833 & 0 & 0.0833 \\ 0 & 0.1667 & 0 \\ 0.0833 & 0 & 0.0833\end{array}\right] \leq \widehat{F}^{\dagger} \widehat{G}=\left[\begin{array}{ccc}0.2826 & 0 & 0.2826 \\ 0 & 0.5652 & 0 \\ 0.2826 & 0 & 0.2826\end{array}\right]$.

Also, for $s(k)=2$, we have

$$
\widehat{P}_{s(k)} \widehat{T}_{s(k)}=\left[\begin{array}{ccc}
1.0719 & 0 & 1.0719 \\
0 & 1.0719 & 0 \\
1.0719 & 0 & 1.0719 \\
2.1438 & 0 & 2.1438
\end{array}\right] \geq 0
$$

Therefore, $\rho\left(\bar{T}_{s(k)}\right)=0.3105 \leq 0.5174=\rho\left(\widehat{T}_{s(k)}\right)<1$.

The nonsingular case is obtained next.

Corollary 3.10 Let $A=U-V$ be a regular splitting of a monotone matrix $A \in$ $\mathbb{R}^{n \times n}$. Let $U=\bar{F}-\bar{G}=\widehat{F}-\widehat{G}$ be weak regular splittings of $U$. If any one of the following conditions hold:

(i) $\quad \bar{F}^{-1} \bar{G} \leq \widehat{F}^{-1} \widehat{G}$ and $\widehat{P}_{s(k)} \widehat{T}_{s(k)} \geq 0$,

(ii) $\quad \bar{F}^{-1} \bar{G} \leq \widehat{F}^{-1} \widehat{G}$ and $\bar{P}_{s(k)} \bar{T}_{s(k)} \geq 0$,

(iii) $\quad \bar{P}_{s(k)} \bar{T}_{s(k)} \leq \widehat{P}_{s(k)} \widehat{T}_{s(k)}$, then $\rho\left(\bar{T}_{s(k)}\right) \leq \rho\left(\widehat{T}_{s(k)}\right)<1$.

The next result shows that the sufficient conditions in Theorem 3.8 can be reduced if we further assume that $U$ is nonnegative.

Theorem 3.11 Let $A=U-V$ be a proper regular splitting of a semimonotone matrix $A \in \mathbb{R}^{m \times n}$. Let $U=\bar{F}-\bar{G}=\widehat{F}-\widehat{G}$ be proper weak regular splittings of a nonnegative matrix $U$. If $\bar{F}^{\dagger} \bar{G} \leq \widehat{F}^{\dagger} \widehat{G}$, then $\rho\left(\bar{T}_{s(k)}\right) \leq \rho\left(\widehat{T}_{s(k)}\right)<1$.

Proof By Theorem 3.2, we have $\rho\left(\bar{T}_{s(k)}\right)<1$ and $\rho\left(\widehat{T}_{s(k)}\right)<1$. Now, using Theorem 3.3, the induced splittings $A=\bar{P}_{s(k)}-\bar{P}_{s(k)} \bar{T}_{s(k)}$ and $A=\widehat{P}_{s(k)}-$ $\widehat{P}_{s(k)} \widehat{T}_{s(k)}$ become proper weak regular. Since $\rho\left(\bar{F}^{\dagger} \bar{G}\right)<1$ and $\rho\left(\widehat{F}^{\dagger} \widehat{G}\right)<1$ as $U$ is semimonotone, the condition $\bar{F}^{\dagger} \bar{G} \leq \widehat{F}^{\dagger} \widehat{G}$ implies that $0 \leq\left[I-\left(\bar{F}^{\dagger} \bar{G}\right)^{s(k)}\right]^{-1} \leq[I-$ $\left.\left(\widehat{F}^{\dagger} \widehat{G}\right)^{s(k)}\right]^{-1}$ which further yields $\bar{P}_{s(k)} \bar{T}_{s(k)}=U\left[I-\left(\bar{F}^{\dagger} \bar{G}\right)^{s(k)}\right]^{-1}-A \leq U[I-$ $\left.\left(\widehat{F}^{\dagger} \widehat{G}\right)^{s(k)}\right]^{-1}-A=\widehat{P}_{s(k)} \widehat{T}_{s(k)}$ as $U$ is nonnegative. Hence, applying Theorem 2.9 
to the splittings $A=\bar{P}_{s(k)}-\bar{P}_{s(k)} \bar{T}_{s(k)}$ and $A=\widehat{P}_{s(k)}-\widehat{P}_{s(k)} \widehat{T}_{s(k)}$, we get $\rho\left(\bar{T}_{s(k)}\right) \leq \rho\left(\widehat{T}_{s(k)}\right)$.

The above result reduces to the following Corollary in the nonsingular matrix setting.

Corollary 3.12 Let $A=U-V$ be a regular splitting of a monotone matrix $A \in$ $\mathbb{R}^{n \times n}$. Let $U=\bar{F}-\bar{G}=\widehat{F}-\widehat{G}$ be weak regular splittings of a nonnegative matrix U. If $\bar{F}^{-1} \bar{G} \leq \widehat{F}^{-1} \widehat{G}$, then $\rho\left(\bar{T}_{s(k)}\right) \leq \rho\left(\widehat{T}_{s(k)}\right)$.

We next provide an example that demonstrates the above result.

Example 3.13 Consider the two-dimensional Laplace's equation

$$
\frac{\partial^{2} u}{\partial x^{2}}+\frac{\partial^{2} u}{\partial y^{2}}=0,0 \leq x \leq 1,0 \leq y \leq 1
$$

with boundary conditions

$$
\left.u(x, y)\right|_{\partial R}=x+y .
$$

Let the square region $R=\{(x, y): 0 \leq x \leq 1,0 \leq y \leq 1\}$ be covered by a grid with sides parallel to the coordinate axis and with equal grid spacing $h=\Delta x=\Delta y$. If $N h=1$, then the number of internal grid points is $(N-1)^{2}$. When $N=6$, the finite difference method using the $\mathcal{O}\left(h^{2}\right)$ central difference discretization on uniform grids generates the linear system $A x=b$, where $A$ is of order 25 and the right hand side vector

$$
b=\left[\frac{1}{3}, \frac{1}{3}, \frac{1}{2}, \frac{2}{3}, 2, \frac{1}{3}, 0,0,0, \frac{4}{3}, \frac{1}{2}, 0,0,0, \frac{3}{2}, \frac{2}{3}, 0,0,0, \frac{5}{3}, 2, \frac{4}{3}, \frac{3}{2}, \frac{5}{3}, \frac{11}{3}\right]^{T}
$$

is derived from Dirichlet boundary conditions. The coefficient matrix $A$ is of the form

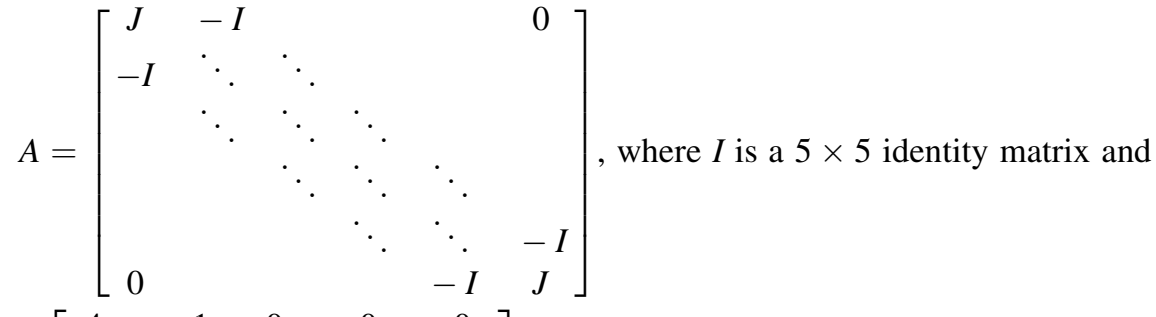

$$
\begin{aligned}
& J=\left[\begin{array}{ccccc}
4 & -1 & 0 & 0 & 0 \\
-1 & 4 & -1 & 0 & 0 \\
0 & -1 & 4 & -1 & 0 \\
0 & 0 & -1 & 4 & -1 \\
0 & 0 & 0 & -1 & 4
\end{array}\right]
\end{aligned}
$$

Setting $U=\operatorname{diag}(A)$, we get a regular splitting $A=U-V$ of the monotone matrix $A$. Further, if $\bar{F}=1.5 U$ and $\widehat{F}=1.75 U$, then $U=\bar{F}-\bar{G}$ and $U=\widehat{F}-\widehat{G}$ 
are two weak regular splittings of the nonnegative matrix $U$. Clearly, $\bar{F}^{-1} \bar{G}=$ $\operatorname{diag}(0.3333,0.3333, \cdots, 0.3333) \leq \widehat{F}^{-1} \widehat{G}=\operatorname{diag}(0.4286,0.4286, \cdots, \quad 0.4286)$. Therefore, for $s(k)=2$, we have $\rho\left(\bar{T}_{s(k)}\right)=0.8809 \leq 0.8906=\rho\left(\widehat{T}_{s(k)}\right)<1$.

The nonnegative restriction on the matrix $U$ in Theorem 3.11 can also be dropped if we replace the condition $\bar{F}^{\dagger} \bar{G} \leq \widehat{F}^{\dagger} \widehat{G}$ by $\left(\bar{F}^{\dagger} \bar{G}\right)^{2} \leq \bar{F}^{\dagger} \bar{G} \leq \widehat{F}^{\dagger} \widehat{G}$, and is proved next.

Theorem 3.14 Let $A=U-V$ be a proper regular splitting of a semimonotone matrix $A \in \mathbb{R}^{m \times n}$. Let $U=\bar{F}-\bar{G}=\widehat{F}-\widehat{G}$ be proper weak regular splittings of U. If $\left(\bar{F}^{\dagger} \bar{G}\right)^{2} \leq \bar{F}^{\dagger} \bar{G} \leq \widehat{F}^{\dagger} \widehat{G}$, then $\rho\left(\bar{T}_{s(k)}\right) \leq \rho\left(\widehat{T}_{s(k)}\right)<1$.

Proof By Theorem 3.4 and the condition $\left(\bar{F}^{\dagger} \bar{G}\right)^{2} \leq \bar{F}^{\dagger} \bar{G}$, the induced splittings $A=\bar{P}_{s(k)}-\bar{P}_{s(k)} \bar{T}_{s(k)}$ and $A=\widehat{P}_{s(k)}-\widehat{P}_{s(k)} \widehat{T}_{s(k)}$ are proper regular and proper weak regular, respectively. Using the inequality $\bar{F}^{\dagger} \bar{G} \leq \widehat{F}^{\dagger} \widehat{G}$, we get $\bar{P}_{s(k)}^{\dagger} \geq \widehat{P}_{s(k)}^{\dagger}$. Thus $\rho\left(\bar{T}_{s(k)}\right) \leq \rho\left(\widehat{T}_{s(k)}\right)$ by Theorem 2.8 .

As a corollary, we have the following result which is new even in the nonsingular matrix setting.

Corollary 3.15 Let $A=U-V$ be a regular splitting of a monotone matrix $A \in \mathbb{R}^{n \times n}$. Let $U=\bar{F}-\bar{G}=\widehat{F}-\widehat{G}$ be weak regular splittings of $U$. If $\left(\bar{F}^{-1} \bar{G}\right)^{2} \leq \bar{F}^{-1} \bar{G} \leq \widehat{F}^{-1} \widehat{G}$, then $\rho\left(\bar{T}_{s(k)}\right) \leq \rho\left(\widehat{T}_{s(k)}\right)<1$.

The next example illustrates the above result.

Example 3.16 Let

$A=\left[\begin{array}{cccccccccc}20 & -0.967 & -1.109 & -0.6948 & -0.2824 & -0.0357 & -1.7314 & -1.654 & -2.4631 & -3.1682 \\ -1.6816 & 20 & -1.1131 & -0.1384 & -1.1361 & -0.3131 & -1.3545 & -0.1639 & -0.7782 & -0.7888 \\ -0.2902 & -0.3625 & 20 & -0.7711 & -0.3427 & -0.1397 & -0.6844 & -0.4231 & -0.1587 & -0.1993 \\ -2.421 & -0.4117 & -0.6286 & 20 & -2.0433 & -0.528 & -0.9069 & -0.449 & -0.9369 & -0.5398 \\ -1.3445 & -1.2228 & -0.0544 & -0.0637 & 20 & -0.7927 & -0.2768 & -0.1316 & -0.1644 & -0.2638 \\ -1.7024 & -0.0895 & -0.4184 & -0.9671 & -1.3149 & 20 & -0.6647 & -0.7289 & -0.4031 & -0.72 \\ -1.3714 & -1.6605 & -0.0633 & -0.2552 & -0.0939 & -0.0451 & 20 & -2.8123 & -2.166 & -3.3831 \\ -1.1986 & -0.4811 & -0.918 & -0.1832 & -0.5206 & -0.1071 & -1.204 & 20 & -0.6027 & -0.4507 \\ -0.7814 & -1.2897 & -0.0222 & -0.3061 & -0.4131 & -0.3528 & -0.7026 & -0.5904 & 20 & -0.4665 \\ -1.8884 & -0.3363 & -0.7448 & -1.0486 & -0.7335 & -0.5682 & -1.5546 & -1.8478 & -2.0934 & 20\end{array}\right]$.

Setting 


$$
U=\left[\begin{array}{cccccccccc}
20 & -0.967 & -1.109 & -0.6948 & -0.2824 & -0.0357 & -1.7314 & -1.654 & -2.4631 & -3.1682 \\
0 & 20 & -1.1131 & -0.1384 & -1.1361 & -0.3131 & -1.3545 & -0.1639 & -0.7782 & -0.7888 \\
0 & 0 & 20 . & -0.7711 & -0.3427 & -0.1397 & -0.6844 & -0.4231 & -0.1587 & -0.1993 \\
0 & 0 & 0 & 20 & -2.0433 & -0.528 & -0.9069 & -0.449 & -0.9369 & -0.5398 \\
0 & 0 & 0 & 0 & 20 & -0.7927 & -0.2768 & -0.1316 & -0.1644 & -0.2638 \\
0 & 0 & 0 & 0 & 0 & 20 & -0.6647 & -0.7289 & -0.4031 & -0.72 \\
0 & 0 & 0 & 0 & 0 & 0 & 20 & -2.8123 & -2.166 & -3.3831 \\
0 & 0 & 0 & 0 & 0 & 0 & 0 & 20 & -0.6027 & -0.4507 \\
0 & 0 & 0 & 0 & 0 & 0 & 0 & 0 & 20 & -0.4665 \\
0 & 0 & 0 & 0 & 0 & 0 & 0 & 0 & 0 & 20
\end{array}\right],
$$

we get a regular splitting $A=U-V$ of the monotone matrix $A$. Further, assuming

$$
\bar{F}=\left[\begin{array}{cccccccccc}
20 & -0.4835 & -0.5545 & -0.3474 & -0.1412 & -0.0179 & -0.8657 & -0.827 & -1.2315 & -1.5841 \\
0 & 20 & -0.5565 & -0.0692 & -0.5681 & -0.1565 & -0.6773 & -0.0819 & -0.3891 & -0.3944 \\
0 & 0 & 20 & -0.3856 & -0.1714 & -0.0698 & -0.3422 & -0.2115 & -0.0794 & -0.0997 \\
0 & 0 & 0 & 20 & -1.0216 & -0.264 & -0.4535 & -0.2245 & -0.4684 & -0.2699 \\
0 & 0 & 0 & 0 & 20 & -0.3963 & -0.1384 & -0.0658 & -0.0822 & -0.1319 \\
0 & 0 & 0 & 0 & 0 & 20 & -0.3323 & -0.3644 & -0.2016 & -0.36 \\
0 & 0 & 0 & 0 & 0 & 0 & 20 & -1.4062 & -1.083 & -1.6916 \\
0 & 0 & 0 & 0 & 0 & 0 & 0 & 20 & -0.3014 & -0.2253 \\
0 & 0 & 0 & 0 & 0 & 0 & 0 & 0 & 20 & -0.2333 \\
0 & 0 & 0 & 0 & 0 & 0 & 0 & 0 & 0 & 20
\end{array}\right]
$$

and

$$
\widehat{F}=\left[\begin{array}{cccccccccc}
20 & -0.0967 & -0.1109 & -0.0695 & -0.0282 & -0.0036 & -0.1731 & -0.1654 & -0.2463 & -0.3168 \\
0 & 20 & -0.1113 & -0.0138 & -0.1136 & -0.0313 & -0.1355 & -0.0164 & -0.0778 & -0.0789 \\
0 & 0 & 20 & -0.0771 & -0.0343 & -0.014 & -0.0684 & -0.0423 & -0.0159 & -0.0199 \\
0 & 0 & 0 & 20 & -0.2043 & -0.0528 & -0.0907 & -0.0045 & -0.0937 & -0.054 \\
0 & 0 & 0 & 0 & 20 & -0.0793 & -0.0277 & -0.0132 & -0.0164 & -0.0264 \\
0 & 0 & 0 & 0 & 0 & 20 & -0.0665 & -0.0729 & -0.0403 & -0.0007 \\
0 & 0 & 0 & 0 & 0 & 0 & 20 & -0.2812 & -0.2166 & -0.3383 \\
0 & 0 & 0 & 0 & 0 & 0 & 0 & 20 & -0.0603 & -0.0451 \\
0 & 0 & 0 & 0 & 0 & 0 & 0 & 0 & 20 & -0.0467 \\
0 & 0 & 0 & 0 & 0 & 0 & 0 & 0 & 0 & 20
\end{array}\right],
$$

we have two weak regular splittings $U=\bar{F}-\bar{G}=\widehat{F}-\widehat{G}$ of $U$. It is easy to observe that $U, \bar{F}$ and $\widehat{F}$ are $M$-matrices and computed as $U=D_{A}+U_{A}, \bar{F}=D_{U}+\frac{1}{2} U_{U}$ and $\widehat{F}=D_{U}+\frac{1}{10} U_{U}$, respectively. Here, $D_{B}$ and $U_{B}$ denote the diagonal and strictly upper triangular entries of the matrix $B$. Clearly,

$$
\left(\bar{F}^{-1} \bar{G}\right)^{2}=\left[\begin{array}{cccccccccc}
0 & 0 & 0.0007 & 0.0006 & 0.0019 & 0.0007 & 0.0019 & 0.004 & 0.0044 & 0.0063 \\
0 & 0 & 0 & 0.0005 & 0.0005 & 0.0007 & 0.0009 & 0.0031 & 0.0025 & 0.004 \\
0 & 0 & 0 & 0 & 0.001 & 0.0005 & 0.0006 & 0.0016 & 0.0017 & 0.0022 \\
0 & 0 & 0 & 0 & 0 & 0.001 & 0.0006 & 0.0021 & 0.0019 & 0.0031 \\
0 & 0 & 0 & 0 & 0 & 0 & 0.0003 & 0.0009 & 0.0007 & 0.0011 \\
0 & 0 & 0 & 0 & 0 & 0 & 0 & 0.0012 & 0.0012 & 0.0018 \\
0 & 0 & 0 & 0 & 0 & 0 & 0 & 0 & 0.0011 & 0.0014 \\
0 & 0 & 0 & 0 & 0 & 0 & 0 & 0 & 0 & 0.0002 \\
0 & 0 & 0 & 0 & 0 & 0 & 0 & 0 & 0 & 0 \\
0 & 0 & 0 & 0 & 0 & 0 & 0 & 0 & 0 & 0
\end{array}\right] \leq
$$




$$
\begin{aligned}
& \bar{F}^{-1} \bar{G}=\left[\begin{array}{cccccccccc}
0 & 0.0242 & 0.0284 & 0.018 & 0.0089 & 0.0016 & 0.0451 & 0.0452 & 0.0658 & 0.0852 \\
0 & 0 & 0.0278 & 0.004 & 0.0288 & 0.0085 & 0.0348 & 0.0071 & 0.0219 & 0.0235 \\
0 & 0 & 0 & 0.0193 & 0.0096 & 0.0039 & 0.0177 & 0.0121 & 0.0056 & 0.0071 \\
0 & 0 & 0 & 0 & 0.0511 & 0.0142 & 0.0233 & 0.0133 & 0.0252 & 0.0165 \\
0 & 0 & 0 & 0 & 0 & 0.0198 & 0.0072 & 0.0042 & 0.0048 & 0.0077 \\
0 & 0 & 0 & 0 & 0 & 0 & 0.0166 & 0.0194 & 0.0113 & 0.0198 \\
0 & 0 & 0 & 0 & 0 & 0 & 0 & 0.0703 & 0.0552 & 0.086 \\
0 & 0 & 0 & 0 & 0 & 0 & 0 & 0 & 0.0151 & 0.0114 \\
0 & 0 & 0 & 0 & 0 & 0 & 0 & 0 & 0 & 0.0117 \\
0 & 0 & 0 & 0 & 0 & 0 & 0 & 0 & 0 & 0
\end{array}\right] \leq \\
& {\left[\begin{array}{cccccccccc}
0 & 0.0435 & 0.0501 & 0.0315 & 0.0134 & 0.0018 & 0.0785 & 0.0758 & 0.1123 & 0.1447 \\
0 & 0 & 0.0501 & 0.0064 & 0.0513 & 0.0143 & 0.0613 & 0.0084 & 0.0358 & 0.0368 \\
0 & 0 & 0 & 0.0347 & 0.0158 & 0.0064 & 0.0310 & 0.0196 & 0.0077 & 0.0097 \\
0 & 0 & 0 & 0 & 0.0919 & 0.0241 & 0.0410 & 0.0209 & 0.0428 & 0.0253 \\
0 & 0 & 0 & 0 & 0 & 0.0357 & 0.0126 & 0.0062 & 0.0076 & 0.0122 \\
0 & 0 & 0 & 0 & 0 & 0 & 0.0299 & 0.0332 & 0.0186 & 0.0330 \\
0 & 0 & 0 & 0 & 0 & 0 & 0 & 0.1266 & 0.0979 & 0.1528 \\
0 & 0 & 0 & 0 & 0 & 0 & 0 & 0 & 0.0271 & 0.0203 \\
0 & 0 & 0 & 0 & 0 & 0 & 0 & 0 & 0 & 0.0210 \\
0 & 0 & 0 & 0 & 0 & 0 & 0 & 0 & 0 & 0
\end{array}\right]=\widehat{F}^{-1} \widehat{G}}
\end{aligned}
$$

Therefore, for $s(k)=2, \rho\left(\bar{T}_{s(k)}\right)=0.2135 \leq 0.2372=\rho\left(\widehat{T}_{s(k)}\right)<1$.

We conclude this section with another comparison result that partially extends Theorem 3.3, [1] to the rectangular case.

Theorem 3.17 Let $A=U-V$ be a proper regular splitting of a semimonotone matrix $A \in \mathbb{R}^{m \times n}$. Let $U=\bar{F}-\bar{G}=\widehat{F}-\widehat{G}$ be proper weak regular splittings of $U$. Consider the iterative scheme defined by (3.1) with corresponding iteration matrices $\bar{T}_{s(k)}$ and $\widehat{T}_{q(k)}$. If the following conditions hold:

(i) $\widehat{P}_{q(k)} \widehat{T}_{q(k)} \geq 0, k=0,1,2, \ldots$,

(ii) $\widehat{F}^{\dagger} \leq \bar{F}^{\dagger}$ and $q(k) \geq s(k), k=0,1,2, \ldots$,

(iii) $\widehat{G} \widehat{F}^{\dagger} \geq 0$, then $\rho\left(\bar{T}_{s(k)}\right) \leq \rho\left(\widehat{T}_{q(k)}\right)<1$.

Proof Since $0 \leq \bar{F}^{\dagger} \bar{G} U^{\dagger}=\bar{F}^{\dagger}(\bar{F}-U) U^{\dagger}=U^{\dagger}-\bar{F}^{\dagger}$, utilizing condition (ii), we get $U^{\dagger}-\bar{F}^{\dagger} \leq U^{\dagger}-\widehat{F}^{\dagger}$. Also, using condition (iii), we observe that $U\left(\widehat{F}^{\dagger} \widehat{G}\right)^{k-1} U^{\dagger}$ $=\widehat{F}\left(I-\widehat{F}^{\dagger} \widehat{G}\right)\left(\widehat{F}^{\dagger} \widehat{G}\right)^{k-1}\left(I-\widehat{F}^{\dagger} \widehat{G}\right)^{-1} \widehat{F}^{\dagger}=\widehat{F}\left(\widehat{F}^{\dagger} \widehat{G}\right)^{k-1} \widehat{F}^{\dagger}=\left(\widehat{G} \widehat{F}^{\dagger}\right)^{k-1} \geq 0$. Now, we will use induction to show that $\left(\bar{F}^{\dagger} \bar{G}\right)^{k} U^{\dagger} \leq\left(\widehat{F}^{\dagger} \widehat{G}\right)^{k} U^{\dagger}, k=0,1,2, \ldots$. For $k=0$, the inequality $\left(\bar{F}^{\dagger} \bar{G}\right)^{k} U^{\dagger} \leq\left(\widehat{F}^{\dagger} \widehat{G}\right)^{k} U^{\dagger}$ is trivial. Suppose that for $k=0,1, \ldots, p$, the inequality holds. Then, for $k=p+1$, we have 


$$
\begin{aligned}
\left(\bar{F}^{\dagger} \bar{G}\right)^{p+1} U^{\dagger} & \leq\left(\bar{F}^{\dagger} \bar{G}\right)\left(\widehat{F}^{\dagger} \widehat{G}\right)^{p} U^{\dagger} \\
& =\bar{F}^{\dagger} \bar{G} U^{\dagger} U\left(\widehat{F}^{\dagger} \widehat{G}\right)^{p} U^{\dagger} \\
& =\left(\bar{F}^{\dagger} \bar{G} U^{\dagger}\right) U\left(\widehat{F}^{\dagger} \widehat{G}\right)^{p} U^{\dagger} \\
& =\left(U^{\dagger}-\bar{F}^{\dagger}\right) U\left(\widehat{F}^{\dagger} \widehat{G}\right)^{p} U^{\dagger} \\
& \leq\left(U^{\dagger}-\widehat{F}^{\dagger}\right) U\left(\widehat{F}^{\dagger} \widehat{G}\right)^{p} U^{\dagger} \\
& =\left(\widehat{F}^{\dagger} \widehat{G} U^{\dagger}\right) U\left(\widehat{F}^{\dagger} \widehat{G}\right)^{p} U^{\dagger} \\
& =\left(\widehat{F}^{\dagger} \widehat{G}\right)^{p+1} U^{\dagger} .
\end{aligned}
$$

Thus $\left(\bar{F}^{\dagger} \bar{G}\right)^{k} U^{\dagger} \leq\left(\widehat{F}^{\dagger} \widehat{G}\right)^{k} U^{\dagger}$ holds for all $k=0,1,2, \ldots$ which implies that $U^{\dagger}-$ $\left(\bar{F}^{\dagger} \bar{G}\right)^{k} U^{\dagger} \geq U^{\dagger}-\left(\widehat{F}^{\dagger} \widehat{G}\right)^{k} U^{\dagger} \quad$ for $\quad$ all $\quad k=0,1,2, \ldots, \quad$ i.e., $\quad \bar{P}_{s(k)}^{\dagger} \geq \widehat{P}_{q(k)}^{\dagger}$, $k=0,1,2, \ldots$. By Theorem 2.8, we thus have $\rho\left(\bar{T}_{s(k)}\right) \leq \rho\left(\widehat{T}_{q(k)}\right)$.

Our next example supports the above theorem.

Example 3.18 Let $A=\left[\begin{array}{ccc}0.25 & 0.25 & 0 \\ 0 & 0 & 0.25 \\ 0.25 & 0.25 & 0 \\ 0 & 0 & 0.25\end{array}\right]$. Then, $A$ is semimonotone. Setting $U=\left[\begin{array}{ccc}0.25 & 0.25 & 0 \\ 0 & 0 & 0.5 \\ 0.25 & 0.25 & 0 \\ 0 & 0 & 0.5\end{array}\right]$, we get a proper regular splitting $A=U-V$ of A. Further, if $\bar{F}=\left[\begin{array}{ccc}0.25 & 0.25 & 0 \\ 0 & 0 & 1 \\ 0.25 & 0.25 & 0 \\ 0 & 0 & 1\end{array}\right]$ and $\widehat{F}=\left[\begin{array}{ccc}0.25 & 0.25 & 0 \\ 0 & 0 & 1.25 \\ 0.25 & 0.25 & 0 \\ 0 & 0 & 1.25\end{array}\right]$, then $U=$ $\bar{F}-\bar{G}=\widehat{F}-\widehat{G}$ are two proper weak regular splittings of $U$. Clearly, $\widehat{F}^{\dagger}=$ $\left[\begin{array}{cccc}1 & 0 & 1 & 0 \\ 1 & 0 & 1 & 0 \\ 0 & 0.4 & 0 & 0.4\end{array}\right] \leq\left[\begin{array}{cccc}1 & 0 & 1 & 0 \\ 1 & 0 & 1 & 0 \\ 0 & 0.5 & 0 & 0.5\end{array}\right]=\bar{F}^{\dagger}$ and $\widehat{G} \widehat{F}^{\dagger}=\left[\begin{array}{cccc}0 & 0 & 0 & 0 \\ 0 & 0.3 & 0 & 0.3 \\ 0 & 0 & 0 & 0 \\ 0 & 0.3 & 0 & 0.3\end{array}\right] \geq 0$. Also, for $q(k)=s(k)=2$, we have $\widehat{P}_{q(k)} \widehat{T}_{q(k)}=\left[\begin{array}{ccc}0 & 0 & 0 \\ 0 & 0 & 0.5313 \\ 0 & 0 & 0 \\ 0 & 0 & 0.5313\end{array}\right] \geq 0$. Therefore, $\rho\left(\bar{T}_{s(k)}\right)=0.625 \leq 0.68=\rho\left(\widehat{T}_{q(k)}\right)<1$. 


\section{Monotone iterations}

In this section, we discuss the monotone convergence theory of the stationary twostage iterative method (3.1) that generalizes Theorem 3.1, [1].

Theorem 4.1 (Monotone Convergence Theorem) Let $A=U-V$ be a proper regular splitting of a semimonotone matrix $A \in \mathbb{R}^{m \times n}$. Let $U=F-G$ be a proper weak regular splitting and $s(k) \geq 1, k=0,1,2 \ldots$ be the inner iteration sequence. If $x_{0}$ and $y_{0}$ are initial values that hold

$$
x_{0} \leq x_{1}, y_{1} \leq y_{0} \text { and } x_{0} \leq A^{\dagger} b \leq y_{0},
$$

then the sequences $\left\{x_{k}\right\}$ and $\left\{y_{k}\right\}$ generated by

$$
\begin{aligned}
& x_{k+1}=T_{s(k)} x_{k}+P_{s(k)}^{\dagger} b, \\
& y_{k+1}=T_{s(k)} y_{k}+P_{s(k)}^{\dagger} b,
\end{aligned}
$$

$k=0,1,2, \ldots$ satisfy

(i) $\quad x_{k} \leq x_{k+1} \leq A^{\dagger} b \leq y_{k+1} \leq y_{k}, k=0,1,2, \ldots$,

(ii) $\lim _{k \rightarrow \infty} x_{k}=A^{\dagger} b=\lim _{k \rightarrow \infty} y_{k}$.

(iii) Further, if $\rho\left(T_{s(k)}\right)<1$, there exist $x_{0}$ and $y_{0}$ which satisfy (4.1).

\section{Proof}

(i) We will show by induction that $x_{k} \leq x_{k+1}$ for $k=0,1,2, \ldots$. The case $k=0$ is established by the hypothesis. Assume that the result holds for $k=0,1 \ldots p>0$ so that $x_{p+1}-x_{p} \geq 0$. Since $s(k)$ is independent of $k$ and $T_{s(k)} \geq 0$ for $k=0,1, \ldots$, we have

$$
\begin{aligned}
T_{s(p)}\left(x_{p+1}-x_{p}\right)= & \left(T_{s(p)} x_{p+1}+\sum_{j=0}^{s(p)-1}\left(F^{\dagger} G\right)^{j} F^{\dagger} b\right)-\left(T_{s(p)} x_{p}\right. \\
& \left.+\sum_{j=0}^{s(p)-1}\left(F^{\dagger} G\right)^{j} F^{\dagger} b\right) \\
= & \left(T_{s(p+1)} x_{p+1}+\sum_{j=0}^{s(p+1)-1}\left(F^{\dagger} G\right)^{j} F^{\dagger} b\right)-\left(T_{s(p)} x_{p}\right. \\
& \left.+\sum_{j=0}^{s(p)-1}\left(F^{\dagger} G\right)^{j} F^{\dagger} b\right) \\
= & x_{p+2}-x_{p+1} \geq 0 .
\end{aligned}
$$

It follows by induction that $x_{p} \leq x_{p+1}$ for each $p$. Similarly, we can show that $y_{p+1} \leq y_{p}$ for each $p$. 
(ii) Applying Lemma 2.3 by considering $K=\mathbb{R}_{+}^{n}$, the nonnegative orthant of $\mathbb{R}^{n}$, the sequences $\left\{x_{k}\right\}$ and $\left\{y_{k}\right\}$ converge to $\left(I-T_{s(k)}\right)^{-1} P_{s(k)}^{\dagger} b$, i.e., $A^{\dagger} b$.

(iii) As $T_{s(k)} \geq 0$, there exists $x \geq 0$ such that $T_{s(k)} x=\rho\left(T_{s(k)}\right) x$ by Theorem 2.1. Since $\rho\left(T_{s(k)}\right)<1$, we have $\rho\left(T_{s(k)}\right) x \leq x$. Let $x_{0}=A^{\dagger} b-x$. Then

$$
\begin{aligned}
x_{1}= & T_{s(k)} x_{0}+\sum_{j=0}^{s(k)-1}\left(F^{\dagger} G\right)^{j} F^{\dagger} b \\
= & T_{s(k)} A^{\dagger} b+\sum_{j=0}^{s(k)-1}\left(F^{\dagger} G\right)^{j} F^{\dagger} b-T_{s(k)} x \\
= & {\left[I-\left(I-\left(F^{\dagger} G\right)^{s(k)}\right)\left(I-U^{\dagger} V\right)\right] A^{\dagger} b+\sum_{j=0}^{s(k)-1}\left(F^{\dagger} G\right)^{j} F^{\dagger} b-\rho\left(T_{s(k)}\right) x } \\
= & {\left[I-\left(I-\left(F^{\dagger} G\right)^{s(k)}\right)\left(I-U^{\dagger} V\right)\right]\left(I-U^{\dagger} V\right)^{-1} U^{\dagger} b } \\
& +\sum_{j=0}^{s(k)-1}\left(F^{\dagger} G\right)^{j} F^{\dagger} b-\rho\left(T_{s(k)}\right) x \\
= & \left(I-U^{\dagger} V\right)^{-1} U^{\dagger} b-\left(I-\left(F^{\dagger} G\right)^{s(k)}\right) U^{\dagger} b \\
& +\sum_{j=0}^{s(k)-1}\left(F^{\dagger} G\right)^{j} F^{\dagger} b-\rho\left(T_{s(k)}\right) x \\
= & \left(I-U^{\dagger} V\right)^{-1} U^{\dagger} b-\sum_{j=0}^{s(k)-1}\left(F^{\dagger} G\right)^{j} F^{\dagger} b \\
& +\sum_{j=0}^{s(k)-1}\left(F^{\dagger} G\right)^{j} F^{\dagger} b-\rho\left(T_{s(k)}\right) x \\
= & A^{\dagger} b-\rho\left(T_{s(k)}\right) x .
\end{aligned}
$$

Similarly, assuming $y_{0}=A^{\dagger} b+x$, and proceeding as above, we get $y_{1} \leq y_{0}$. Moreover, $y_{0}-x_{0}=2 x \geq 0$ which implies $y_{0} \geq x_{0}$.

Note that condition (iii) in Theorem 4.1 guarantees the existence of $x_{0}$ and $y_{0}$ which satisfy the inequality (4.1). We conclude the paper with the remark that the convergence theory of two-stage iteration scheme for type II splittings even in the nonsingular matrix setting is not yet established in the literature. Very recently, Shekhar et. al. [23] introduced convergence theory of type II double splittings. Motivated by the same work, we plan to study the problem of the convergence of the two-stage iteration scheme for type II splittings near future.

Acknowledgements We thank the referee for the constructive and insightful comments, which have helped us to substantially improve our manuscript. We thank the Government of India for introducing the work from home initiative during the COVID-19 pandemic. 
Funding This study was partially funded by Science and Engineering Research Board, New Delhi, India, under the Grant number MTR/2017/000174. The last author acknowledges the support.

\section{Compliance with ethical standards}

Conflicts of interest The authors declare that they have no conflict of interest.

Human participants This article does not contain any studies with human participants or animals performed by the authors.

\section{References}

1. Bai, Z.-Z., and D.-R. Wang. 1997. The monotone convergence of the two-stage iterative method for solving large sparse systems of linear equations. Applied Mathematics Letters 10: 113-117.

2. Ben-Israel, A., and T.N.E. Greville. 2003. Generalized Inverses. Theory and Applications. New York: Springer.

3. Berman, A., and M. Neumann. 1976. Proper splittings of rectangular matrices. SIAM Journal on Applied Mathematics 31: 307-312.

4. Berman, A., and R.J. Plemmons. 1974. Cones and iterative methods for best least squares solutions of linear systems. SIAM Journal of Numerical Analysis 11: 145-154.

5. Berman, A., and R.J. Plemmons. 1994. Nonnegative matrices in the mathematical sciences. Philadelphia: SIAM.

6. Cao, Z.-H. 2001a. Convergence of block two-stage iterative methods for symmetric positive definite systems. Numerische Mathematik 90: 47-63.

7. Cao, Z.-H., and D.J. Evans. 2001. Convergence of two-stage iterative methods for singular symmetric positive semidefinite systems. International Journal of Computer Mathematics 76: 363-374.

8. Cao, Z.-H. 2001b. On the convergence of nonstationary iterative methods for symmetric positive (semi)definite systems. Applied Numerical Mathematics 37: 319-330.

9. Climent, J.-J., Devesa, A., and Perea, C. 2000. Convergence results for proper splittings. In N. Mastorakis (ed.) Recent Advances in Applied and Theoretical Mathematics (pp. 39-44). World Scientific, Singapore.

10. Climent, J.-J., and C. Perea. 2003. Iterative methods for least-square problems based on proper splittings. Journal of Computational and Applied Mathematics 158: 43-48.

11. Frommer, A., and B. Szyld. 1992. H-splitting and two-stage iterative methods. Numerische Mathematik 63: 345-356.

12. Golub, G. 1965. Numerical methods for solving linear least squares problem. Numerische Mathematik 7: 206-216.

13. Greville, T.N.E. 1966. Note on the generalized inverse of a matrix product. SIAM Review 8: 518-521.

14. Jena, L., D. Mishra, and S. Pani. 2014. Convergence and comparison theorems for single and double decomposition of rectangular matrices. Calcolo 51: 141-149.

15. Lanzkron, P.J., D.J. Rose, and D.B. Szyld. 1991. Convergence of nested classical iterative methods for linear systems. Numerische Mathematik 58: 685-702.

16. Liu, Z.-Y., H.-B. Wu, and L. Lu. 2000. The two-stage iterative methods for symmetric positive definite matrices. Applied Mathematics and Computation 114: 1-12.

17. Migallón, V., and J. Penadés. 1999. The monotonicity of two-stage iterative scheme. Applied Mathematics Letters 12: 73-76.

18. Mishra, D. 2017. Further study of alternating iterations for rectangular matrices. Linear Multilinear Algebra 65: 1566-1580.

19. Mishra, D., and K.C. Sivakumar. 2012. On splitting of matrices and nonnegative generalized inverses. Operators and Matrices 6: 85-95.

20. Mishra, N., and D. Mishra. 2018. Two-stage iterations based on composite splittings for rectangular linear systems. Computers and Mathematics with Applications 75: 2746-2756. 
21. Nichols, N.K. 1973. On the convergence of two-stage iterative processes for solving linear equations. SIAM Journal on Numerical Analysis 10: 460-469.

22. Plemmons, R.J. 1976. Regular splittings and the discrete Neumann problem. Numerische Mathematik 25: $153-161$.

23. Shekhar, V., C.K. Giri, and D. Mishra. 2020. A note on double weak splittings of type II. Linear Multilinear Algebra. https://doi.org/10.1080/03081087.2020.1795057.

24. Varga, R.S. 2000. Matrix Iterative Analysis. Berlin: Springer.

25. Wang, C.L., and J.H. Zhao. 2005. Further results on regular splittings and multisplittings. International Journal of Computer Mathematics 82: 421-431.

26. Wang, L. 2007. Semiconvergence of two-stage iterative methods for singular linear systems. Linear Algebra and its Applications 422: 824-838.

Publisher's Note Springer Nature remains neutral with regard to jurisdictional claims in published maps and institutional affiliations. 